\title{
Leonardo da Vinci-The First Bioengineer: Educational Innovation to Meet His Desire for Knowledge and Promote His Concept of Interdisciplinarity
}

\author{
Ricardo Luis Armentano, Luis Kun \\ Department of Biological Engineering, Universidad de la República, Montevideo, República Oriental del Uruguay \\ Email: armen@ieee.org
}

How to cite this paper: Armentano, R. L., \& Kun, L. (2019). Leonardo da Vinci-The First Bioengineer: Educational Innovation to Meet His Desire for Knowledge and Promote His Concept of Interdisciplinarity. Creative Education, 10, 1180-1191. https://doi.org/10.4236/ce.2019.106089

Received: May 6, 2019

Accepted: June 21, 2019

Published: June 24, 2019

\section{Copyright $\odot 2019$ by author(s) and} Scientific Research Publishing Inc. This work is licensed under the Creative Commons Attribution International License (CC BY 4.0).

http://creativecommons.org/licenses/by/4.0/

\section{(c) (i) Open Access}

\begin{abstract}
The central axis of the work has been to empower the figure of Leonardo da Vinci 500 years after his death. With him, we wanted to infect our students with their tireless desire to know and learn. We plant the possibility of carrying out a project of educational innovation giving a push to the process of teaching and learning by making it more participatory, cooperative and interdisciplinary, as well as the desire to involve more the students encouraging their interest in learning and work. To honor Leonardo's legacy and the spirit of the Renaissance, it is necessary to teach to stimulate the critical spirit and to encourage imagination and creativity. Leonardo da Vinci was a prolific hybrid innovator to unlock the secrets of human complexity for the first time in the history of mankind amalgamating Science, Engineering, Biology and Engineering; so, we define him as the first bioengineer of humanity.
\end{abstract}

\section{Keywords}

Creativity, Interoperability, Humanism, Engineering Education, Teaching for Creativity, Innovation

\section{Introduction}

The figure of Leonardo da Vinci 500 years after his death continues to illustrate his tireless desire to know and learn. Leonardo is the symbol of a century in which progress impacted, shattering the thickness of dogmas. In the Quattrocento, the doors have definitely opened, the ideas have spread and still nourish us, clear our path and enlighten us. The spirit of the Renaissance (Burckhardt, 1878) teaches us fervor, admiration, the strength of the critical spirit and trust in 
man, and often reveals that even without realizing, there are obscurantisms that constantly threaten man and that, even perennial, lie in ambush. Florence, in Leonardo's time, was the Silicon Valley of the Renaissance (Isaccson, 2017). The famous Florentine House of the Medici, along with other similar families, financed thinkers and creators from different fields (artists, scientists, poets and philosophers, among others), achieved an interdisciplinary exchange of ideas (Burke, 1999). The writer Frans Johansson called this phenomenon the Medici Effect (Johansson, 2006), which boomed a great explosion of creativity in fifteenth century Italy, whose basic idea is that when you "access an intersection of fields, disciplines or cultures, you can combine concepts existing in a large number of new ideas". Leonardo summed up the Renaissance ideal of the multitalented sage-painter, sculptor, narrator, musician, scientist, mathematician, architect, engineer with a thirst for insatiable knowledge and curiosity (Bortolon, 1967).

From a reigning culture, most students do not learn, as they only memorize equations, data and procedures. The core part is that students learn to manipulate symbols, but do not know what it means. They are not being taught to think, but to pass the test. It is postulated that this is one of the most important reasons for the crisis that science schools in general and engineering schools in particular are going through-low level of interest and problems of recruitment and retention of students. In effect, the traditional structure of engineering presents characteristics that encourage abandonment. First, knowledge is presented in isolation in each discipline in completely independent courses, which is a serious impediment to future multidisciplinary work. On the other hand, students lack purpose, not being clear about the usefulness of the basic knowledge they receive and being unaware of the tools and habitual ways of working in their future profession (Armentano, 2012). This work develops the framework of the Degree in Biological Engineering, which is a novel engineering training with solid knowledge of biological processes and life sciences, in order to generate professionals capable of industrial development and develop the necessary equipment to medicine and for the biotechnological and agri-food industries (Kun, 2012).

The objective of this work has been to empower the figure of Leonardo da Vinci in the 500 years death anniversary. With him, we wanted to infect our students with their tireless desire to know and learn. We plant the possibility of carrying out a project of educational innovation giving a push to the process of teaching and learning by making it more participatory, cooperative and interdisciplinary, as well as the desire to involve more the students encouraging their interest in learning and work.

\section{Backgrounds}

Leonardo who was born on April 15, 1452 at his father's place in Anchiano, a village in the small town of Vinci, summed up the Renaissance ideal of the sage of multiple talents-painter, sculptor, narrator, musician, scientist, mathemati- 
cian, architect, engineer-with a thirst for insatiable knowledge and curiosity (Isaccson, 2017). Leonardo died in France in 1519 and is buried in the Château d'Amboise in the Loire Valley. The last three years of his life were spent in the service of the king of France, Francis I (Isaccson, 2017).

Natural son of a small notary of Florence, at the age of eighteen, he mastered huge knowledge-mathematics, physics, chemistry and anatomy. Music, too, has no secrets for him (Isaccson, 2017). His strange genius directs him to all fields of knowledge and works that today we would call "engineering". Very early, he reveals himself as a universal spirit, his vision of the world is that of a visionary in the real world. Leonardo laid the foundations of new experimental sciences; it moved away from mere empiricism towards concepts of the science of applied mechanics suitable for general application in the industrial world, in that sense It was the first modern engineer Leonardo had less influence because his writings did not have enough diffusion and some were lost. What would have happened if Galileo and Kepler had known them? Leonardo loves the four profession deserves not so much for being a practicing engineer but as a prophet of the future of engineering and the more his legacy is studied more this great dimension of great Florentine understood (Birkett, 2016).

Leonardo's contributions to physiology flourished in the late 15th century long before the scientists that we often think of as the founding fathers of our discipline. These include Boyle, Hooke, Malpighi, Priestley, and Lavoisier. All of these were working more than 150 yrs. after Leonardo. He also has the distinction of being the first bioengineer, that is, applying the principles of engineering to physiology (West, 2017; Sterpetti, 2019a). Leonardo's great innovation was to take well-established concepts and subject them to the direct verification of phenomena, always using his great capacity for observation, equipped with his extraordinary ability to transform what he saw into a graphic schematic of processes. In a certain sense, Leonardo da Vinci is somehow out-of-this-world (Isaccson, 2017).

Leonardo has anticipated and foreshadowed with his research phenomena of great current ecology, nuclear destruction, the use of science for war purposes, with the limited resources available at that time. His most precious legacy is not the 21 paintings or the almost 100,000 drawings and sketches he left, but his creation of a new way of thinking. On the other hand, their ability to interconnect the various fields of knowledge, increasing tenfold their cognitive potential when intertwines n, is a good example of "interdisciplinarity" (to use a modern expression) constitutes a fundamental basis for bioengineers of the present (Valentinuzzi \& Pallotti, 2013). But it goes far beyond these specific innovations. $\mathrm{He}$ points out in his Codes of hypotheses that science will take several centuries to verify. Intuitions that surprise us with their conceptual power and prophetic precision (Birkett, 2016).

Leonardo was the most visionary of all the engineers (Birkett, 2016). Water is "the blood of the Earth," he writes, the main and essential element of the cycle in which all forms of life are articulated. How did he come to such a conclusion? It 
is always he who affirms that man is "composed of earth, water, air and fire, like the earthly body (...)". That all its cellular metabolism, an idea in itself totally unthinkable at the time, is based on aqueous exchanges.

It is without a doubt the first of all ecologists! He senses that the water cycle, both on our entire planet and on the very basis of any living organism, must be based on an unalterable equilibrium without which we run the risk of a disaster (Kirby et al., 1990).

In the house of Florence, which lives modestly in the shadow of the splendor of the palaces, in the heart of the night, a description of a distant world is made to come. For himself, because the Church does not like the creators of prophecies that escaped his control.

It predicts a world in which this fundamental cycle of vital water that the Apocalypse will threaten will be disturbed. A world where we have totally ignored the deep reality of life. A world where the discoveries of science, while they will be dazzling, will be applied in disorder (Isaccson, 2017).

Since his sixteenth century Florence, Leonardo has seen the great polluted cities of our time, he perceived the poisoning of the air, the nauseating and dangerous substances that kill the oceans. And the war ... a terrible war, of flame and steel, a war of which he speaks even in terms of "rupture of matter in the most intimate elements ...". Is it not the division of the atom?

Leonardo does not ignore, in fact, that the technique of which he is one of the most brilliant pioneers in history could be at the origin of a disaster if it were pushed to the extreme and used in a frantic way. Whenever he feels it is too early to give his contemporaries an idea or similar device, such or such a mechanism, he abstains. Never a useless vanity, the desire to be recognized and celebrated motivated him. He prefers to look sadly at the diagrams and descriptions of his discoveries. At the risk of sometimes displeasing their powerful protectors) (Isaccson, 2017).

Leonardo theorized that diseases derived from some imperfection in the structure of the human body (Sterpetti, 2019b). He put in evidence that for each disease atherosclerosis, pathology of cardiac valves, cirrhosis and portal hypertension, chronic obstructive pulmonary disease amongst others, there was a correlated anatomic change, overcoming the old theories of the diseases as unbalance of the humor of the body (Sterpetti, 2019b).

Leonardo studied the dynamics of water flow in rivers, using colors to show flow patterns, defining the voltage on the side walls of the river. It determined with different colors flow characteristics in the center and near the edges of rivers and translate these findings to the blood flowing in arteries. Leonardo received permission to dissect cadavers at the Santa Maria Nuova Hospital in Florence (Kerr, 1957). The notes of his diaries include detailed studies of the human skull, human fetus, internal organs of a woman, muscles, tendons, skeletal, cardiovascular system. Leonardo moved slowly inside the accepted theories he learned from reading, but adapted to the new ideas, based on their own experiments. The complexity of the study of the human body by the current bioengi- 
neers follow the steps of Leonardo due to they addresses many systems that interact in complex and nonlinear ways. Physiological systems of human beings interact jointly at all times to allow the body to continually adapt to the conditions imposed leads to the concept of complexity to advance the understanding of deep phenomena. Leonardo laid the first foundations of that understanding. This work deals about Leonardo da Vinci who was a prolific hybrid innovation to unlock the secrets of human complexity for the first time in the history so we define him as the first bioengineer of humanity

\section{Development of the Experience}

In the strictly methodological aspect, it is proposed to make an introductory course more interactive than the normal conference-type course where the classmates become professors (Armentano, 2012) encourage the interest of our students. It is fundamental about teaching by asking not giving the answer because the important thing is not the answer but the reasoning to reach it. It seeks to teach students and students to THINK. Frequently the effort to retain the Master's words conspires against analysis, logic, and reasoning. A university education that does not stimulate the critical spirit and that does not teach to think is not higher education but a training to the submission. Any person trained in a scientific education cannot be sensitive to sectarian propaganda or intolerance of any kind whether religious, political or administrative (Armentano \& Kun, 2016).

The structure of the course seeks to cover the relevant topics of Biological Engineering and provides the basis for graduates to be astute doers, reliable innovators, agents of change, main integrators, business facilitators, technology administrators and knowledge repositories insisting that transformative changes require a joint effort due to the enormous complexity of the interdisciplinary activity (Kun, 2009), assuming that individual efforts are often insufficient to overcome the challenges encountered in the classroom and in the changing environments labor (Cymberknop \& Armentano, 2016).

The idea is that the academic body and students are put under appropriate pressure and temperature levels incorporating an imprint, that acts as an important catalyst in this reaction, assuming individual and institutional maturity to judge and appreciate a culture that celebrates the rarity, the capacity of choice, the independence, the entrepreneurial spirit, concentration, creativity and intensity. This culture is on the other hand the great challenge of the University: to ensure a microclimate where the best science and technology can be exercised and at the same time to lead its students to the full development of their potential as people, as citizens and as workers; to put ideas into action; generating new understanding concomitant to the new technological utilities, paradigm of research oriented to innovation; for the good of society and to improve the lives of people with continuous progress in quality, cost reduction and preservation of the environment (Armentano, 2012). 
This is a mandatory course for the Freshman students. The course provides 16 credits. It's a semester-long course. It's a mandatory course and does not have prerequisites (it is the first introductory course in the career). The course faculty consists of one full professor, and 4 teaching assistants (including a medical doctor). The teaching method is a blended learning, including classroom lectures, interactive quizzes, and presentations. The students are assessed based on their performance and participation in the activities during the course. However, the key evaluation is through a final poster presentation at the end of the course, where the students choose an important topic of their field of biological engineering, perform a thorough literature review, and present in the form of a poster. The students exposed the results they had investigated, achieving that classmates become professors. A considerable improvement was evident along the course rising the percentage of students expressing willingness to teach interdisciplinary lessons in the future (Gero, 2017).

To develop this project, we set ourselves a series of objectives:

- Encourage the interest of our students for a period (the Renaissance) characterized by the desire for knowledge, highlighting the figure of Leonardo as a prototype of the time.

- Create a humanistic environment that promotes the existence of points of view reciprocal and favor the acceptance of the other, according to their customs and culture.

- Deal in a globalized way with different contents of different areas with the purpose of improving teaching and learning processes.

- Incorporate reading and writing as forms of personal enrichment of the students.

- Encourage reflection and cooperative work of the educational team.

- Search interdisciplinary resources to develop the teaching process learning.

Activities carried out to encourage the interest of our students:

1) Who was Leonardo da Vinci?

Specific objective: we try to offer the most human aspects of Leonardo's scientific, technical and artistic work, thus avoiding the danger of mythologizing the geniuses.

2) Engineering is the engine of innovation

Specific objective: Concepts of innovative research in modern engineering abandoning the old basic vs. applied science paradigm.

3) The body as a signal generator

Specific objective: to take signals from different places of the human body in a non-invasive way. A Physiology laboratory where students act as subjects, making innovative physiological measurements with non-invasive and safe means, all under a scientific and safe methodology.

4) Learning to Innovate in Biological Engineering

Specific objective: simulations of research through examples where creativity and innovation are the transversal axes.

List of the talks delivers where the classmates become professors in order to encourage reflection and innovative works: 
Themes developed in the framework of this academic year 2018

- Health and technology are biocompatible.

- Description of the growth of sheep by mathematical models.

- Optogenetics: Illuminate your mind.

- Biomechanical model of the trachea for the diagnosis of Parkinson's disease.

- Advance and uses of electronic skin.

- Monitoring of the brain to improve quality of life.

- Prosthetics "Low Cost".

- Wearable Microfluidic device for the capture, storage and colorimetric analysis of sweat.

- Nanotechnology: the hope of oncology.

- Re-stabilizing the rhythm: Automatic external defibrillators available to everyone.

- Application of Artificial Intelligence to the estimation of heart rate from facial videos.

- Spider silk: "The material of the future".

- Brain-computer interface.

- Diapers inspire a new way of studying the brain.

- Otoacoustic Emissions. The power of a neonatal diagnosis.

Themes developed in the framework of this project year 2017

- Retinal prosthetics and intraocular implants to correct blindness.

- Application of thermography in the diagnosis of breast cancer.

- Molecular imaging of breasts.

- White blood hypertension of Bata Blanca and its detection.

- Mapping of the brain: research processes and importance in the scientific and health field.

- Transcranial Magnetic Stimulation.

- Bioinformatics at the service of pharmacovigilance.

- Artificial Heart. Ventricular assist devices.

- Da Vinci Surgery Surgical System.

- Brain/computer interface and neuroprosthetics applied to quadriplegic people.

- Robotic surgery: its development and evolution.

- Bionic Kidney.

- Bionic Engineering: a new life opportunity.

- Cyborg Beast: The low-cost prosthetics for children.

- 3D printer as a tool in medicine.

- Print 3D ovaries and nano-robots to combat infertility.

- GPS technology in sport.

- Purification of ambient air.

- Graphene: the material of the future.

\section{Discussion}

Today, definitely, Leonardo would have used electronic technologies and com- 
puters. But probably he would continue to bet on the continuous torque of the scientific and artistic dipole, which could be a special key for the advancement of knowledge. Its integral and ubiquitous approach to the planet could be the precedent of the phrase "Think globally, act locally" used for the first time in the context of environmental challenges, has taken a more widespread value and use in recent years (Kun, 2009). Education under the leitmotiv Think Globally, Act Locally is one of the most powerful and important things in our lives and should be taught to young students. Because everything is connected, although disconnected (Kun, 2010). This disconnection has a high cost, and a look into the future predicts that most serious issues will be related to population growth and demographics and will be due to the needs of water, food, medicine and energy, for example, availability, production, demand, distribution, price, etc. (Armentano \& Kun, 2016). The solution to these situations or circumstances requires a holistic approach, (Kun, 2014) consequence of globalization, for example, the global economy, and above all, the encouragement of collaboration between industry, academia, the hospital environment, laboratories and government for bringing together experts from different disciplines to investigate and meet the specific needs of a product or service with high added value. The leitmotiv of the new engineering is that size the power of creativity; audacity and adventure to discover and learn from change. The great challenge of our times is to stimulate creativity, discarding the myth that affirms that it is something magical, a natural power denied to the majority of mortals and given to only a few and that it remains for them to imagine what never existed before. Creativity is not magic, nor an inherent attribute of our genes, much less a supernatural blessing. It is a capacity, and anyone can learn to be creative and get the most out of it. To provoke creativity, few things are as important as the time devoted to cross-fertilization with fields different from our areas of specialization: great advances often depend on the naive audacity of a layman (Armentano, 2012).

To honor Leonardo's legacy we seek to re-find Leonardo's creative spirit, curiosity and imagination, sheltered by an area where artists, scientists, poets and philosophers, among others, achieve an interdisciplinary exchange of ideas, products, goods and services prioritizing the link from academia to work to improve productivity by empowering science, technology and innovation as engines of economic growth and human development. We look for the academia-industry-hospital clinics interaction, to generate sufficient resources and obtain modern facilities in order to make more attractive a university career for the new generations, and in this way ignite the interest to complete the university studies. We encourage the installation of laboratories with advanced technology to improve the quality of education in engineering and science and create opportunities for thousands of creative young people to contribute to the innovation process. Certainly, government commitment and assistance are essential to help create and maintain the necessary ecosystem. In this global context, and based on innovative strategic thinking, we must teach thinking to connect ideas 
and disruptive discoveries in an ecosystem that stimulates a critical mind refractory to sectarian propaganda, or any kind of intolerance: religious, political, social, cultural, economic or administrative (Armentano, 2012).

On the basis of Leonardo's legacy and the spirit of the Renaissance, it is necessary to teach to think about the framework of the new planetary Trinity that governs our days: globalization, westernization and development. It is to stimulate the critical spirit and teach to apprehend as an antidote to the factory model education that is a training for submission. It is connecting ideas and disruptive discoveries in an ecosystem that stimulates a critical mind refractory to sectarian propaganda, or any kind of intolerance: religious, political, social, cultural, economic or administrative. It is to encourage innovation and creativity as a way to apprehend learning, entrepreneurship, communication and sharing. It is to train young people in interdisciplinary and functional interoperability to avoid adopting solutions based on partial knowledge of the problems, which prevents achieving real solutions to complex problems (Kun, 2018). It is stimulating to think of the Italian Renaissance as a form of intellectual revolution in an unstable environment, where criticism of the old theories represented was not only an offense to tradition, but also a threat to established power. Vinci appears in this paradoxical movement as a beacon on this night, a tenacious and stable brightness; the genius of all geniuses. Leonardo knows of the hostilities engendered by all novelty, the panic that any questioning of the established world can unleash. He possesses the unbridled strength of freedom that man must always conquer, tenaciously maintaining the creative ardor and the fierce need to know, not to leave the world as it is, but to change it. It was not easy for Leonardo to express his bold ideas freely in the tumultuous environment of the Italian Renaissance and that is why his figures come. His life is still a great puzzle that has not yet been fitted, and there are still many things to discover. It would take immense erudition. There are thousands of pamphlets written from right to left, blueprints, sketches, diagrams, sketches that sleep in several large libraries of the world. No one has dared to bring them together to make a vast synthesis of this enormous material in suspense that one does not stop wondering how a man, who is also one of the greatest universal painters, has been able to accumulate it during a simple life. It is possible that Leonardo, for the oneiric dimension of his research, still surpasses us.

As we celebrate 500 years of Da Vinci's life it is worth connecting all those outcomes from his incredible life, to who this man actually was and how he was able to achieve everything he did. According to multiple sources and his biography Leonardo was an illegitimate child, dyslexic, illiterate, bipolar and with attention deficit disorder. It is obvious then that his curiosity, his observation capacity, his sacrifice and particularly his perseverance driven by his passion account for his successes, regardless of the amount of times that he failed trying. According to Richard Branson, "bipolar" is not simply a "disorder". It is a symptom of high levels of creativity and energy; and it is found in most geniuses, 
i.e., Isaac Newton, Van Gogh, Michelangelo, etc. Hyper focus is a natural asset of bipolar disorder when it's aroused. Leonardo's testament is a refreshing reminder to the human race, that despite the difficulties that he encountered and that humans may face throughout a lifetime, the important thing is to keep trying. Later, the accomplishments achieved can eclipse forever the failures experienced along the way. We are reminded a quote by Einstein: "Imagination is more important than Knowledge" (Maxwell, 2003). Leonardo da Vinci's imagination was extraordinary and so were the talents that he developed throughout his life. One of Leonardo's most important lessons is that failure and how we handle failure is what forms us as humans. A hybrid is a combined product of elements of different nature. Leonardo was a true hybrid in all senses. And that's very important because he obtained a solid training that had enabled him to address things that did not exist in the society in which he lived.

Ecosystems that foster creativity and innovation will be those who will ensure the development of these innovative environments, which was already a reality for at least 500 years in Florence Renaissance. Also in the Montmartre of the 20 where Picasso, Modigliani, and a host of creators who made a quantum change in the arts and in the Greenwich Village 60 with Bod Dylan as flagship made ecosystems that fostered creativity. We must turn this explosion of creative ideas and expose ourselves to the intersection of fields as individuals, teams or organizations. These fields can be disciplines, cultures, domains in which one specializes through education, work, hobbies, traditions and life experiences. Today we can see similar cases in large innovation centers like Silicon Valley and Israel as start-up nation.

Dr. Rene Favaloro, the famous Argentine cardiovascular surgeon who revolutionized the field with his pioneering contributions, among which the most outstanding was the standardization of bypass surgery coronary artery, first performed in 1967, mentored us on the road of hybrid engineering (Armentano \& Kun, 2016). It was located in the techno-space between biology, medicine and engineering, made by those paired with creative thinking and interoperable strategies, becoming a facilitator of technological innovation with the scientific Medici effect. Dr. Favaloro held and nurtured an interdisciplinary group of young students, engineers, doctors, physicists, physiologists, veterinarians, mathematicians and other specialists under appropriate levels of pressure and temperature.

\section{Conclusion}

Leonardo was an example of hybridization, and at the time it was shown that if you put people to work together in the same discipline, will be adding knowledge, but instead if people from different disciplines come together, rather than adding, what is being done is multiplying the probabilities that the result of the joint work is innovative. Leonardo da Vinci was a prolific hybrid innovation to unlock the secrets of human complexity for the first time in the history of man- 
kind amalgamating Science, Engineering, Biology and Engineering; so we define him as the first bioengineer of humanity.

Our advice to young people is to look for connections where you never thought existed, and to integrate and innovate. Take a step back and see the big picture and remember that simplicity yields complexity. To honor Leonardo's legacy and the spirit of the Renaissance, it is necessary to teach to stimulate the critical spirit and to encourage imagination and creativity. It is connecting ideas and disruptive discoveries in an ecosystem that stimulates a critical mind refractory to sectarian propaganda, or any kind of intolerance: religious, political, social, cultural, economic or administrative.

\section{Acknowledgements}

The authors gratefully acknowledge Parag Chatterjee for his helpful contributions revising the manuscript.

\section{Conflicts of Interest}

The authors declare no conflicts of interest regarding the publication of this paper.

\section{References}

Armentano, R. L. (2012). Updating Engineering Education in the Southern Cone: Creativity and Innovation. Creative Education, 3, 733-736. https://doi.org/10.4236/ce.2012.326109

Armentano, R., \& Kun, L. (2016). Upgrading Translational Engineering in Medicine and Biology through Conscious-Technology with Humanistic Motivation and Global Vision. Health and Technology, 6, 27-34. https://doi.org/10.1007/s12553-016-0125-7

Birkett, D. (2016). Leonardo: Artist \& Engineer. Engineering \& Technology, 11, 68-71. https://doi.org/10.1049/et.2016.0110

Bortolon, L. (1967). The Life and Times of Leonardo. London: Paul Hamlin.

Burckhardt, J. (1878). The Civilization of Renaissance in Italy. Middlemore: Trans S.G.C.

Burke, P. (1999). The Italian Renaissance: Culture and Society in Italy. Princeton, NJ: Princeton University Press.

Cymberknop, L. J., \& Armentano, R. L. (2016). Global View and Future Challenges of Biomedical Engineering in Latin-America: Experts' Opinion. Health and Technology, 6, 205-211. https://doi.org/10.1007/s12553-016-0144-4

Gero, A. (2017). Students' Attitudes towards Interdisciplinary Education: A Course on Interdisciplinary Aspects of Science and Engineering Education. European Journal of Engineering Education, 42, 260-270. https://doi.org/10.1080/03043797.2016.1158789

Isaacson, W. (2017). Leonardo Da Vinci. New York: Simon \& Schuster.

Johansson, F. (2006). The Medici Effect: What Elephants and Epidemics Can Teach Us about Innovation. Boston, MA: Harvard Business School Press.

Kerr, J. M. (1957). William Hunter. His Life, Personality and Achievements. Scottish Medical Journal, 2, 372-378. https://doi.org/10.1177/003693305700200907

Kirby, R., Withington, S., Burr Darling, A., \& Gridley Kilgour, F. (1990). Engineering in History. New York: Dover Publications Inc. 
Kun, L. (2009). Inaugural Keynote: Interoperability Vision Needed to Transform US Public Health. In Health Care Reform or Transformation: An Interoperability Summit. Washington DC: National Academy of Science. https://www.hawaii.edu/csati/a/agenda.html

Kun, L. (2009). Invited Closing Keynote: Biomedical Engineering and Global Health Transformation: A Vision of Opportunities and Challenges. In Argentine Biomedical Engineering Society (SABI) Annual Meeting.

Kun, L. (2010). Opening Lecture: Merging Medical Humanism and Technology. In Annual International Conference of the IEEE Engineering in Medicine and Biology, Buenos Aires, 31 August-4 September 2010. https://doi.org/10.1109/IEMBS.2010.5626557

Kun, L. (2012). Invited Inaugural Keynote to Kick-Off the Start of a New Career in "Biological Engineering”. In 21st Century: Importance of Science and Technology Education to the Development of a Nation. Paysandu, Uruguay: CenUR Litoral Norte Universidad de la Republica. http://www.cup.edu.uy/index.php/dep-extension.html

Kun, L. (2014). Invited Lecture: Convergence of Communications, Informatics and Biology with Public Health: A Holistic and Interoperable Systems Approach for Healthcare Transformation. In Proceedings of CLAIB 2014, VI Latin American Congress on Biomedical Engineering CLAIB 2014, Parana, Entre Rios, Argentina,

Kun, L. (2018). Invited Speaker: Creating a Culture of Collaboration: Inspire, Innovate \& Participate in Consensus Standards Development. In 2018 IEEE Life Sciences Conference (LSC 2018), Montreal.

Maxwell, J. C. (2003). Thinkings for a Change: 11 Ways Highly Successful People Approach Life and Work. New York: Warner Business Books.

Sterpetti, A. (2019a). Cardiovascular Research by Leonardo da Vinci (1452-1519). Circulation Research, 124, 189-191. https://doi.org/10.1161/CIRCRESAHA.118.314253

Sterpetti, A. (2019b). Cardiovascular Physio-Pathology by Leonardo Da Vinci (1452-1519). Circulation Research, 124, 472-474. https://doi.org/10.1161/CIRCRESAHA.118.314390

Valentinuzzi, M., \& Pallotti, G. (2013). Leonardo the Bioengineer Retrospectroscope. IEEE Pulse, 4, 58-62. https://doi.org/10.1109/MPUL.2013.2271417

West, J. B. (2017). Leonardo da Vinci: Engineer, Bioengineer, Anatomist, and Artist. American Journal of Physiology-Lung Cellular and Molecular Physiology, 312, L392-L397. https://doi.org/10.1152/ajplung.00378.2016 